\title{
Time-dependent efficiency measurements of polymer solar cells with dye additives: unexpected initial increase of efficiency
}

\author{
Kyle J. Bandaccari ${ }^{1}$, Grace E. Chesmore ${ }^{2,3}$, Mitchel Bugaj ${ }^{2}$, Parisa Tajalli-Tehrani Valverde ${ }^{1}$, \\ Richard P. Barber Jr. ${ }^{2,3}$, and Brian J. McNelis ${ }^{1, *}$ \\ ${ }^{1}$ Department of Chemistry and Biochemistry, Santa Clara University, Santa Clara, CA 95053, USA \\ 2 Department of Physics, Santa Clara University, Santa Clara, CA 95053, USA \\ ${ }^{3}$ Center for Nanostructures, Santa Clara University, Santa Clara, CA 95053, USA
}

Received: 13 July 2017 / Received in final form: 20 January 2018 / Accepted: 24 January 2018

\begin{abstract}
We report the effects of the addition of two azo-dye additives on the time-dependent efficiency of polymer solar cells. Although the maximum efficiencies of devices containing different amounts of dye do not vary greatly over the selected concentration range, the time dependence results reveal a surprising initial increase in efficiency in some samples. We observe this effect to be correlated with a leakage current, although a specific mechanism is not yet identified. We also present the measured lifetimes of these solar cells, and find that variations in dye concentrations produce a small effect at most. Characterization of the bulk heterojunction layer (active layer) morphology using atomic-force microscope (AFM) imaging reveals reordering patterns which suggest that the primary effects of the dyes arise via structural, not absorptive, characteristics.
\end{abstract}

Keywords: polymer photovoltaic / dye additives / morphology / leakage current

\section{Introduction}

Third-generation, solution-processed solar cells represent the forefront of research in solar technology. Since the first observation of a photoactive polymer engaging in an electron transfer process [1], polymer solar cells (PSCs) have garnered substantial attention in the subfield of organic photovoltaics [2]. In the years since, the emergence of numerous device architectures has resulted in incremental efficiency increases with [3,4] highest reported efficiencies for PSCs (9\%) [5] approaching but not yet competitive with current high-performing commercial silicon solar cells.

Even with lower efficiencies, PSCs possess two distinct manufacturing advantages. Conventional inorganic solar cells base their photoactive layers upon crystalline semiconductor-grade silicon, a material which requires the use of harsh and hazardous etchants such as hydrofluoric acid and handling within a strict cleanroom environment to prevent contamination during processing. In contrast, the active layers of PSCs may be applied in solution [4]. PSCs, therefore, may be produced much more safely, quickly, and easily than their silicon-based counterparts in methods which require fewer specialized apparatuses.

\footnotetext{
* e-mail: bmcnelis@scu.edu
}

As a second advantage, PSCs may be produced in mechanically flexible forms via roll-to-roll processes on existing organic light-emitting diode equipment [6,7]. The resulting PSCs have thinner active layers, meaning that they use less material and result in thinner, lighter, cheaper, and more durable units when produced at scale.

Despite the promise of PSCs, the technology does suffer from key drawbacks in both efficiency and lifetime. Silicon solar cells still possess higher efficiencies overall, and they last much longer when their active layers are properly encapsulated.

Although extensive work has been undertaken in pursuit of achieving increased efficiency for PSCs, there has been relatively little focus on characterizing and improving their long-term stability. This work aims to contribute to the body of knowledge concerning the longterm function of PSCs by providing comparative studies of device lifetime patterns and mechanisms through analysis of electrical and morphological data collected in ambient conditions. Despite the comparatively low efficiency marks we generate, our lifetime data is generated from transport measurements spanning multiple orders of magnitude. Additionally, measuring in ambient conditions condenses what would be thousands of hours of lifetime testing to a matter of days to enable the characterization of a large number of devices. 


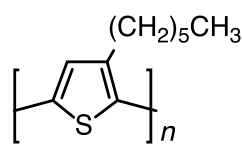

P3HT

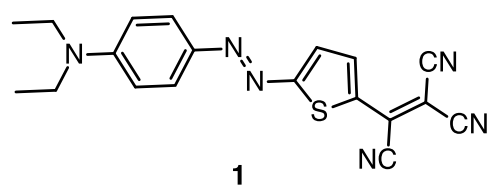

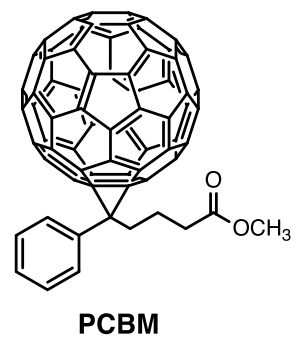

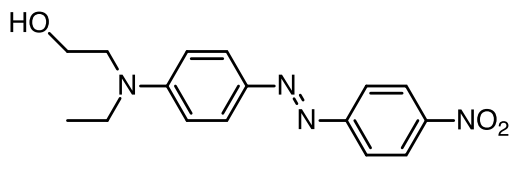

DR1

Fig. 1. Poly(3-hexylthiophene-2,5-diyl) (P3HT), [6,6]-phenyl $\mathrm{C}_{61}$ butyric acid methyl ester (PCBM) and the two dye additives examined in this study: 1 and Disperse Red 1 (DR1).

This study expands upon our prior work on measuring the lifetime responses generated by altering the composition of devices containing the well-studied combination of poly(3-hexylthiophene-2,5-diyl) (P3HT) and $[6,6]$-phenyl $\mathrm{C}_{61}$ butyric acid methyl ester (PCBM) (Fig. 1). Previously, we reported differing efficiency and lifetime behaviors for polymer-based devices in which we replaced PCBM with one of a small library of PCBM derivatives $[8,9]$.

In this work, we examined the affect a third active layer component in the form of one of two dye additives. Interestingly, phthalocyanine dyes have been reported in fullerene-P3HT devices, and donor-acceptor dyes based on a diketopyrrolopyrrole core and a cyanovinylene-4nitrophenyl acceptor have also been shown to increase device efficiency [10-13]. In addition, a simple donoracceptor dye was covalently bonded to form a novel PCBM ester which nearly doubled device efficiency [14]. Donor-acceptor dye additives have also been used in dye sensitized solar cells to set a record efficiency for those devices at the time, and an indane group dye has also been shown to produce some efficiency improvements $[15,16]$.

We chose two dyes; the first is a novel synthetic green dye, $\mathbf{1}[17]$ and the second is a commercially-available red dye, Disperse Red 1 (DR1) (Fig. 1). The addition of other light-absorbing components has been shown to improve PSC efficiency [18], and their inclusion here was undertaken with the same intent in a comparative approach for two structurally similar dyes with non-overlapping absorbance characteristics. Both $\mathbf{1}$ and DR1 are azo dyes, which in our earlier work had shown excellent properties and photochemical stability. Previous work has included dyes in low concentrations with the intent of harvesting these charge localizations using the polymer/fullerene system both as independent additives $[11,13,19-22]$ and as reactive products of PCBM itself [14]. We also examined device lifetime, which is an important criteria for viable, commercial devices.

\section{Experimental}

All solution-processing steps were carried out within a glovebox under $\mathrm{N}_{2}$ atmosphere with $\mathrm{O}_{2}$ concentration of $<25 \mathrm{ppm}$ and $\mathrm{H}_{2} \mathrm{O}$ concentration of $<1 \mathrm{ppm}$. The desired amounts of solid PCBM ester ( $>99.0 \%$, American Dye Source), P3HT (regioregular, Sigma-Aldrich), 1, and DR1 were separately placed in tared $3 \mathrm{~mL}$ vials. All compounds except 1 were used as received. Chlorobenzene (99.9\%, Sigma-Alrdrich) was then added as needed to prepare solutions at approximately $1.5 \mathrm{wt} . \%$. The vials were sealed and the solutions were stirred via magnetic stirrer on a hotplate for a minimum of $24 \mathrm{~h}$ with gentle heating $\left(40^{\circ} \mathrm{C}\right)$ to achieve complete dissolution. A 1:1 wt.:wt. mixture of P3HT:PCBM (molar fraction of PCBM $x=0.16$ ) [9] at these concentrations was then prepared by mixing aliquots of the solutions and repeating the $24 \mathrm{~h}$ stirring at $40^{\circ} \mathrm{C}$ to ensure the preparation of a homogeneous solution. After cooling to room temperature, aliquots of the previously prepared PCBM:P3HT mixture were used to prepare various dye solutions ranging from 2 to $10 \mathrm{wt} . \%$ with respect to $\mathrm{PCBM}$.

Indium tin oxide (ITO)-coated glass substrates (8$12 \Omega /$ sq., Delta Technologies) were patterned in acid (37\% $\mathrm{HCl}$, Sigma-Aldrich) using an acrylic coating to serve as a mask. The substrates were immersed for $15 \mathrm{~min}$ and then rinsed with deionized water before undergoing sonication for $10 \mathrm{~min}$ successively in acetone and 2-propanol and drying under a stream of compressed air. The patterned substrates were then subjected to plasma cleaning at a base pressure of $300 \mathrm{mtorr}$ for $10 \mathrm{~min}$. and then immediately transferred to an $\mathrm{N}_{2}$-containing glovebox and spin-coated with poly(3,4-ethylenedioxythiophene):poly(styrenesulfonate) (PEDOT:PSS, 1.3 wt.\% in $\mathrm{H}_{2} \mathrm{O}$, conductive grade, Sigma-Aldrich). The PEDOT:PSS coated substrates were then heated to evaporate solvent at $200^{\circ} \mathrm{C}$ for $2 \mathrm{~h}$.

Once at room temperature, the active layer mixtures were applied via the same spin coating process. The active layer coating on the substrates was then annealed at $190^{\circ} \mathrm{C}$ 


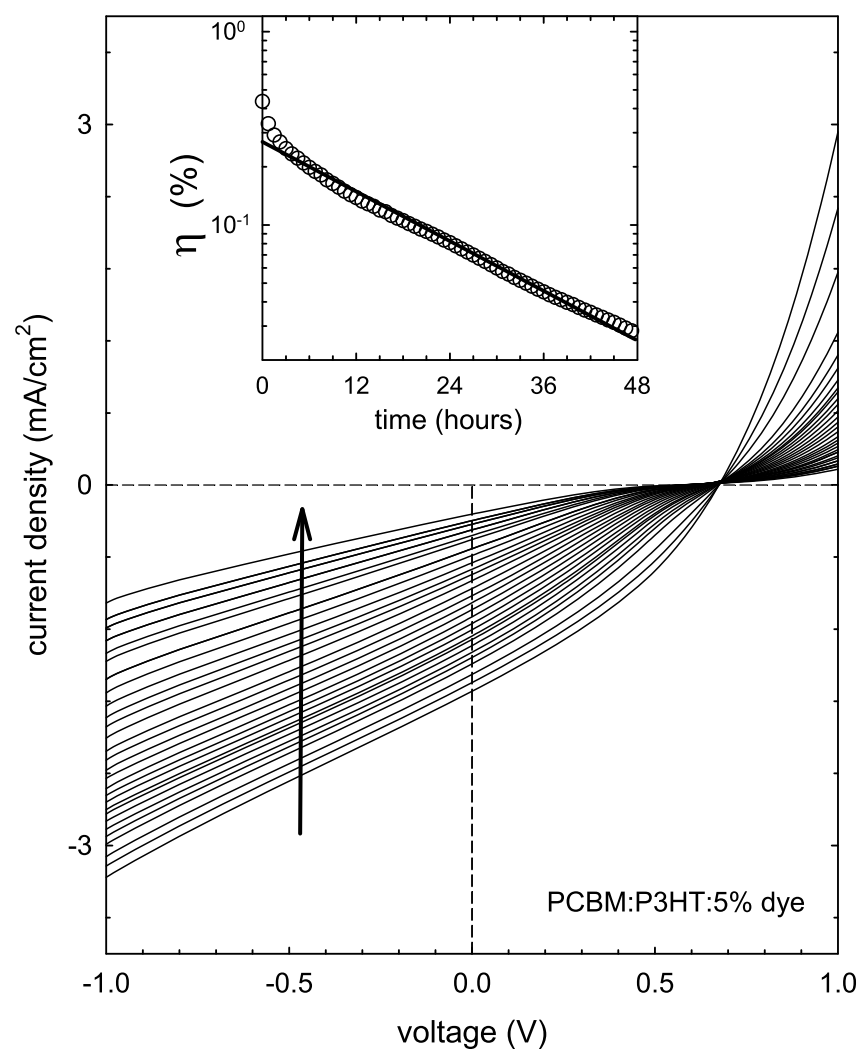

Fig. 2. Current-voltage (I-V) curves that exhibit the degradation of a PCBM:P3HT:dye device in ambient conditions. The arrow indicates the progression of time. Inset: the power conversion efficiency of this device in a semilog plot as a function of time. The solid line fit is used to extract the lifetime $\tau$ (reciprocal of the slope).

for $1 \mathrm{~h}$. To complete fabrication, samples were then transported inside an airtight vessel carrying a dry $\mathrm{N}_{2}$ atmosphere to a bell-jar evaporator system. Samples were immediately transferred and placed under vacuum for an atmospheric exposure time of less than $5 \mathrm{~min}$. The final layers consisting of $1 \mathrm{~nm}$ of $\mathrm{LiF}$ and $100 \mathrm{~nm}$ of $\mathrm{Al}$ were evaporated through a shadow mask onto the substrates at a base pressure of $5 \mu$ torr. The resulting samples contained six devices each with individual areas of approximately $0.25 \mathrm{~cm}^{2}$.

Subsequent electrical measurements were taken in ambient atmosphere immediately following sample preparation. Current-voltage (I-V) curves were measured in alternating dark and illuminated conditions under a PV Measurements, Inc. Small-Area Class-B Solar Simulator. Data collection was automated with a custom MATLAB script to control an IEEE 488 Bus interfaced Keithley 2400 SourceMeter. Samples remained in the low-light conditions of the laboratory environment throughout testing under an indirectly-controlled temperature of $23-26^{\circ} \mathrm{C}$ and a relative humidity of $50-70 \%$.

For certain active layer solutions, atomic force microscopy (AFM) was used to characterize the surface morphology. Sample substrates were prepared in the same manner as the ITO slides, but uncoated glass microscope

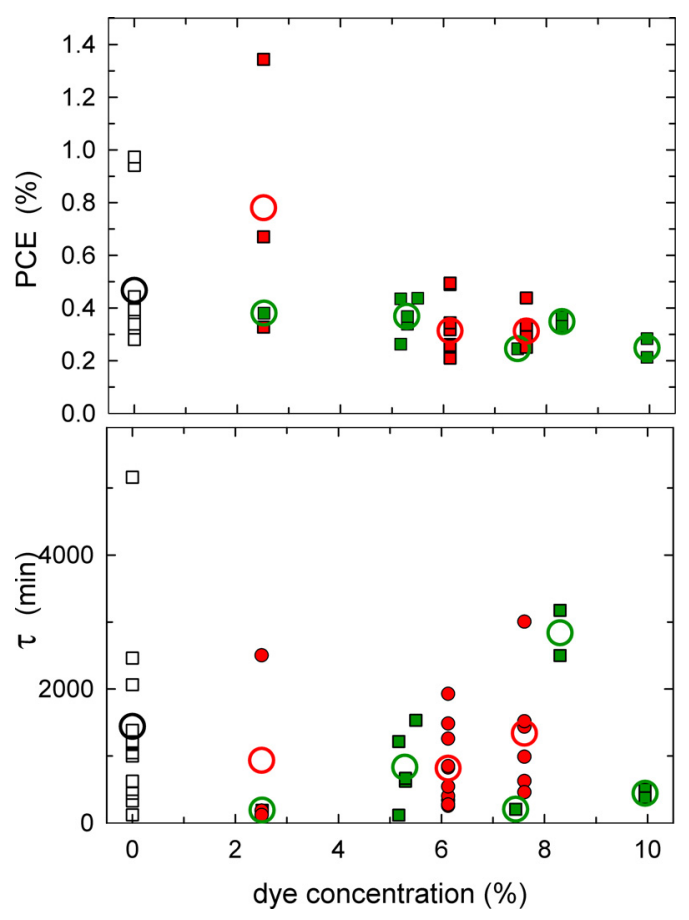

Fig. 3. Efficiency and lifetime data from a series of PCBM: P3HT:dye devices. We plot: (top) the initial power conversion efficiency $\eta$; and (bottom) the decay time constant $\tau$ as a function of dye weight percent with respect to PCBM. The small symbols refer to each sample measured and the large circles show the average value for each dye concentration. Black symbols represent samples without dye whereas the red and green symbols refer to red (DR1) and green (1) dyes respectively.

slides were substituted. Samples were spin-coated with PEDOT:PSS and the active layer solution and subsequently annealed using the same procedure described earlier. AFM imaging was conducted on a Veeco Dimension 3100 atomic force microscope operated in tapping mode.

\section{Results and discussion}

Time-dependent efficiency measurements are standard procedure for our group. Given the observation that most devices undergo an exponential decay in $\eta$ with time, these data have allowed us to define a characteristic lifetime for each sample $(\tau)$. In other words most samples show a timedependent efficiency that behaves as

$$
\eta(t)=\eta(0) e^{-t / \tau}
$$

This behavior is shown in Figure 2 and its inset. Typically, the $\eta$ values corresponding to successive IV curves (where the arrow denotes the progression of time) decay exponentially and monotonically as shown in the inset. The initial $\eta$ value is calculated from the first IV curve, and the slope of the linear fit on the semilog axis yields the value of $\tau$. A summary of these two parameters for all devices measured is presented in Figure 3. 


\section{Compound 1}

$5 \%$

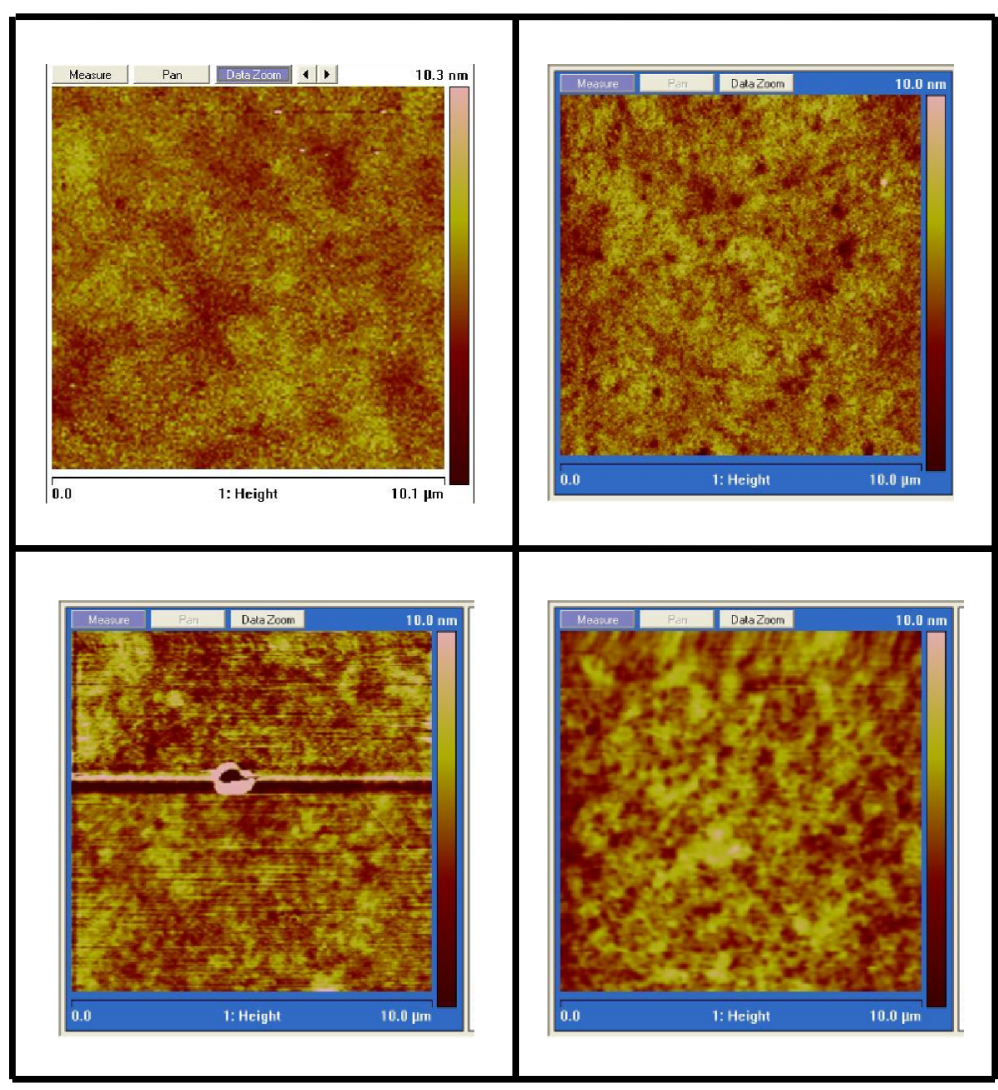

\section{DR1}

Fig. 4. Atomic force microscope images the reveal the surface morphology of the active layers without Al contacts. The leftmost frame shows a PCBM:P3HT sample without dye. The two columns show the effects of green dye (1, top row) and red dye (DR1, bottom row) at $5 \%$ and $10 \%$ weight concentrations with respect to the PCBM content. The addition of both dyes clearly enhances aggregation in the samples.

From this collection of data, it is intriguing that many of the devices with the highest efficiencies include a low concentration of dye additive. Intuitively, the addition of a high concentration of a strong chromophore should enhance the absorptive properties of the active layer and increase the overall efficiency of the device. Instead, it appears that the layer is saturated by the dye additives, even at these seemingly low concentrations. The wavelength of maximum absorbance $\left(\lambda_{\max }\right)$ is $719 \mathrm{~nm}$ for $\mathbf{1}$ [17] and $502 \mathrm{~nm}$ for DR1 [23]. Competing absorption at these wavelengths could explain the efficiency decreases that are observed at higher dye concentrations within our range when compared to control devices. The lack of statistically significant differences in lifetime and efficiency values for dye-containing devices suggests that the effects of the dyes are related to $\eta$ or $\tau$ through means which are not immediately apparent.

Imaging studies revealed an effect of the dye additives, which could not be extracted from the summarized data. Figure 4 presents a series of representative AFM images of active layers. The electrical and absorbance data are insufficient to conclusively determine the primary effects of $\mathbf{1}$ and DR1 in our polymer devices. This is because it has been shown that both fullerenes and P3HT form aggregates in solution and in thin-film devices [24-32], and that morphological ordering determines the quality of a device. The control image shows the fine grain structure associated with neat layers, but regional differences accumulate once dye is introduced. From these images, the lack of difference from baseline efficiencies of $5 \mathrm{wt} . \%$ (and lower) $\mathbf{1}$ and DR1 devices are qualified by the fine-grain morphological ordering they share with the unmodified P3HT:PCBM layer.

The 10 wt.\% samples especially showcase aggregation not observed in those with lower concentrations of dye. These images show larger features or aggregations on the order of $2-5 \mu \mathrm{m}$. At $10 \mathrm{wt} . \%$, the layers even display pinholes. When taken in conjunction with the transport results presented above, the onset of morphological reordering at similar concentrations suggests that the dyes exert their effects by means of their structural properties instead of their absorption characteristics. A similar result was observed when PCBM was substituted with a longer chain version PCBOD (PCBM ester). Spectroscopic data 


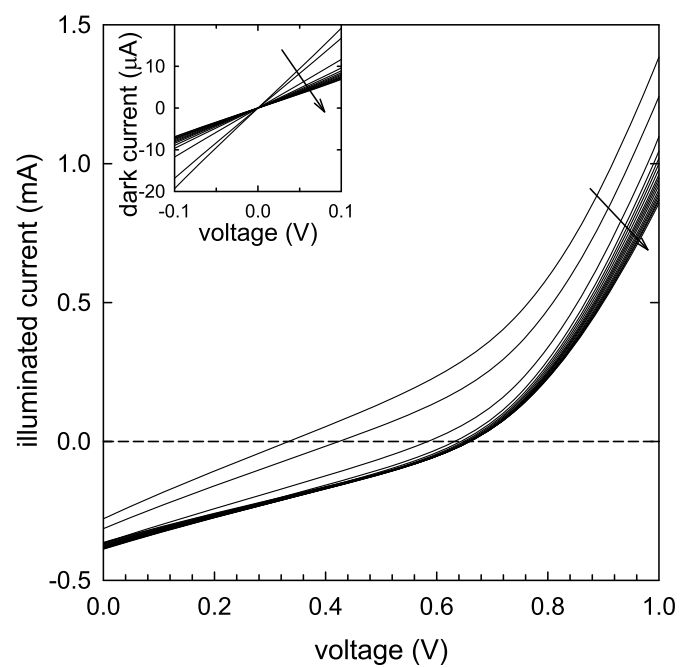

Fig. 5. Current-voltage (I-V) curves showing an initial increase in both open-circuit voltage and short-circuit current (main frame). The inset shows the corresponding zero-bias I-V curves while the sample is in the dark. The progression of time is indicated by the arrows.

suggested that the inclusion of this more soluble fullerene somehow disrupted the crystallinity of the P3HT thereby degraded the efficiencies of those devices [9]. A recent study used 1,8 diiodooctane as an alternate solvent to control active layer aggregation [33]. In this case, exposure to light appeared to alter the chemistry of the layer as well, through the formation of radicals. We observed clear morphology effects of our dye additives, however direct chemical effect were beyond the scope of our measurements but are unlikely to occur by a similar mechanism.

Upon initial review, efficiency decay appears consistent for all devices; however, we occasionally observed efficiencies with non-monotonic time dependence. In particular, some devices showed $\eta$ initially increasing before the onset of exponential decay. Similar behavior has been observed in previous studies, $[9,34]$ and was tentatively linked to an additional conductance through the devices [34]. In the current data set we identified six samples with similar behavior. Given the occasional unexpected $\eta$ measurement due to bad electrical contacts, we chose only those devices which had at least two $\eta$ values below and before the peak value. We modeled these $\eta(t)$ curves as an exponential decay (for long times) with an additional efficiency suppression that typically disappeared between one and two hours after the sample measurements were started. Figure 5 shows IV curves for such a sample. The arrow denotes the direction of time, and the efficiency clearly increases at first. Although it is somewhat more difficult to make out the longer time behavior (curves become overlapping), Figure 6 reveals the beginning of exponential decay in $\eta$ around $100 \mathrm{~min}$ (shown with the solid line fit to the efficiency data). Figure 5 (inset) also displays the IV curves near the origin under dark conditions. We denote the slope of these IV curves as the dark zero-bias conductance $\left(G_{\text {dark }}\right)$. The time evolution of $G_{\text {dark }}$ is also displayed on Figure 6.

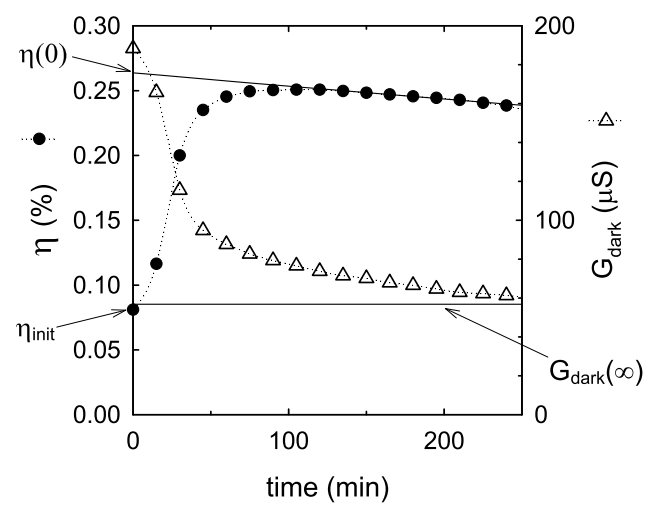

Fig. 6. The power conversion efficiency (filled circles) and the zero-bias dark conductance (open triangles) of one sample as a function of time. We note the clear correlation between the decrease in conductance and the increase in efficiency. Denoted on the figure are the symbol that represents the initial efficiency $\left(\eta_{\text {init }}\right)$, the extrapolated point on the y-axis that represents the $t=0$ efficiency $(\eta(0))$ and the line representing the asymptotic conductance value after long times $\left(\mathrm{G}_{\text {dark }}(\infty)\right)$; these values are explained in the discussion.

It is apparent in Figure 6 that the decrease in $G_{\text {dark }}$ corresponds to $\eta(t)$ rising to a peak value before beginning an exponential decay. This behavior suggests that the excess conduction seen in $G_{\text {dark }}$ partially shunts current back through the device thereby robbing it of some efficiency. As $G_{\text {dark }}$ drops $\eta$ increases. In order to compare this effect across multiple samples we characterize the suppression of $\eta$ as $\Delta \eta=\eta(0)-\eta_{\text {init }}$, where $\eta_{\text {init }}$ is the initial measured value of $\eta$ (shown on Fig. 6) and $\eta(0)$ is the value from the expected exponential decay, $\eta(t)=\eta(0) e^{t / \tau}$. In other words $\eta(0)$ is the expected value of $\eta$ at $t=0$ if the suppression had been removed. To compare samples of different efficiencies we then normalize the suppression by $\eta(0)$ and compare $\frac{\Delta \eta}{\eta(0)}$ to the change in $G_{\text {dark, }}$ given by $\Delta G_{\text {dark }}=G_{\text {dark }}(0)-$ $G_{\text {dark }}(\infty)$. Note that $G_{\text {dark }}(0)$ is the initial measured $G_{\text {dark }}$ and $G_{\text {dark }}(\infty)$ is the value after a long time (denoted on Fig. 6). Figure 7 shows the six cases from the current study (solid squares). The good correlation suggests that this plot is a reasonable way to visualize that larger conductance changes correlate with larger initial suppression of $\eta$. The linear regression fit is based on the six PCBM/dye:P3HT samples.

As noted previously, we had observed a similar effect in our published data [9]. Using the same criteria we identified four more samples of PCBOD:P3HT and have plotted them here (open triangles). Note that the four additional results are the product of a different study with a different fullerene ester and no dye additive. We find the agreement with the current data to be remarkable. This result suggests that our approach is a valid way to not only visualize the current data but also to compare different systems. Although we have not identified a physical mechanism for the shunt or leakage current that suppresses the efficiency in these devices, we have confidence that the dark conductance measurement 


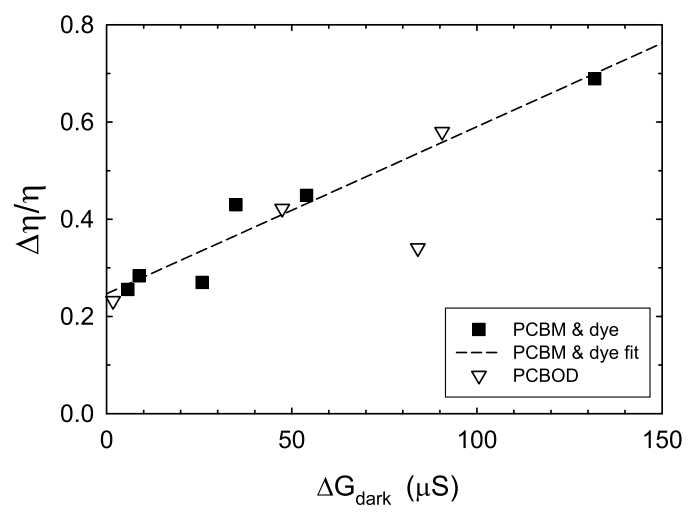

Fig. 7. Plot of the relative change in efficiency with respect to the change in zero-bias dark conductance of six samples from the current dye additive work (filled squares). The dashed line shows a fit to these same points. The open triangles represent the same analysis performed on four previous samples using a modified fullerene ester (PCBOD). Note that these four values were not part of the fit, suggesting that this behavior might be somewhat universal.

is a valid way to characterize it. One possible explanation relates to the indane dye study mentioned previously [16]. They used poling during device fabrication to organize the polar dye molecules yielding performance enhancements in the resulting solar cell. In our devices, at the beginning of irradiation an electric field develops in operating device which could promote a favorable migration and organization of device components such as the dye and/or fullerene molecules, which enhances the performance of the device at early time points. Fullerene migration in layered devices is well documented in the literature [35], and we found that slowing this migration can dramatically improve device lifetimes [36]. At initial times, this migration could yield constructive changes in molecule aggregation similar to the poling fabrication study.

\section{Conclusion}

The results of these studies with PSCs exhibit the extraordinary need for continued work towards understanding the patterns and mechanisms which drive the efficiency and especially the lifetime characteristics of all types of experimental solar cells. Our two series with dye additives exemplify how little is known about even subtle modifications to device architecture. Despite the relatively low efficiencies we achieve and the small concentration range of dye chosen, the data still show a great amount of experimental resolution. Small changes in the identity and amount of dye additives exert morphological changes within the active layer which influence the parameters that determine the performance of devices. Additionally, thorough lifetime analyses provide crucial information and a more complete understanding of device characteristics and function than simple measurements of initial efficiency can offer.
We acknowledge G. Sloan for invaluable technical assistance. Funding was provided by a Santa Clara University (SCU) Sustainability Grant, SCU Miller Center Roelandts Grants, a Rich Bastiani Research Award, the SCU Office of Student Fellowships and a Clare Boothe Luce Scholar Award.

\section{Author contribution statement}

K.J. Bandaccari conducted experimental work and data analysis and contributed to the writing of the manuscript G.E. Chesmore, M. Bugaj, and P. Tajalli-Tehrani Valverde conducted experimental work and data analysis R.P. Barber and B.J. McNelis directed the research as co-principle investigators and contributed to the writing of the manuscript and preparation of the figures.

\section{References}

1. N.S. Sariciftci, L. Smilowitz, A.J. Heeger, F. Wudl, Photoinduced electron transfer from a conducting polymer to buckminsterfullerene, Science 258, 1474 (1992)

2. N.S. Sariciftci, D. Braun, C. Zhang, et al., Semiconducting polymer-buckminsterfullerene heterojunctions: diodes, photodiodes, and photovoltaic cells, Appl. Phys. Lett. 62, 585 (1993)

3. S.E. Shaheen, C.J. Brabec, N.S. Sariciftci, F. Padinger, T. Fromherz, J.C. Hummelen, $2.5 \%$ efficient organic plastic solar cells, Appl. Phys. Lett. 78, 841 (2001)

4. G. Li, V. Shrotriya, J. Huang, et al., High-efficiency solution processable polymer photovoltaic cells by self-organization of polymer blends, Nat. Mater. 4, 864 (2005)

5. X. Guo, N. Zhou, S.J. Lou, et al., Polymer solar cells with enhanced fill factors, Nat. Photonics 7, 825 (2013)

6. F.C. Krebs, S.A. Gevorgyan, J. Alstrup, A roll-to-roll process to flexible polymer solar cells: model studies, manufacture and operational stability studies, J. Mater. Chem. 19, 5442 (2009)

7. F.C. Krebs, S.A. Gevorgyan, B. Gholamkhass, et al., A round robin study of flexible large-area roll-to-roll processed polymer solar cell modules, Sol. Energy. Mater. Sol. Cells 93, 1968 (2009)

8. B.H. Johnson, E. Allagoa, R.L. Thomas, et al., Influence of functionalized fullerene structure on polymer photovoltaic degradation, Sol. Energy Mater. Sol. Cells 94, 537 (2010)

9. E.L. Sena, J.H. Peel, D. Wesenberg, et al., Transport and spectroscopic studies of the effects of fullerene structure on the efficiency and lifetime of polythiophene-based solar cells, Sol. Energy Mater. Sol. Cells 100, 192 (2012)

10. S. Honda, T. Nogami, H. Ohkita, H. Benten, S. Ito, Improvement of the light-harvesting efficiency in polymer/ fullerene bulk heterojunction solar cells by interfacial dye modification, ACS Appl. Mater. Interfaces 1, 804 (2009)

11. S. Honda, H. Ohkita, H. Benten, S. Ito, Multi-colored dye sensitization of polymer/fullerene bulk heterojunction solar cells, Chem. Commun. Camb. Engl. 46, 6596 (2010)

12. S. Honda, H. Ohkita, H. Benten, S. Ito, Selective dye loading at the heterojunction in polymer/fullerene solar cells, Adv. Energy Mater. 1, 588 (2011)

13. G.D. Sharma, S.P. Singh, M.S. Roy, J.A. Mikroyannidis, Solution processed bulk heterojunction polymer solar cells with low band gap DPP-CN small molecule sensitizer, Org. Electron. 13, 1756 (2012) 
14. J.A. Mikroyannidis, A.N. Kabanakis, S.S. Sharma, G.D. Sharma, A simple and effective modification of PCBM for use as an electron acceptor in efficient bulk heterojunction solar cells, Adv. Funct. Mater. 21, 746 (2011)

15. N. Cai, S.-J. Moon, L. Cevey-Ha, et al., An organic D- $\pi$-A dye for record efficiency solid-state sensitized heterojunction solar cells, Nano Lett. 11, 1452 (2011)

16. L. Sicot, C. Fiorini, A. Lorin, P. Raimond, C. Sentein, J.-M. Nunzi, Improvement of the photovoltaic properties of polythiophene-based cells, Sol. Energy Mater. Sol. Cells 63, 49 (2000)

17. C.R. Moylan, B.J. McNelis, L.C. Nathan, M.A. Marques, E. L. Hermstad, B.A. Brichler, Challenging the auxiliary donor effect on molecular hyperpolarizability in thiophene-containing non-linear chromophores: X-ray crystallographic and optical measurements on two new isomeric chromophores, J. Org. Chem. 69, 8239 (2004)

18. M. Grätzel, Dye-sensitized solar cells, J. Photochem. Photobiol. C Photochem. Rev. 4, 145 (2003)

19. Y.-S. Yen, W.-T. Chen, C.-Y. Hsu, H.-H. Chou, J.T. Lin, M.C.P. Yeh, Arylamine-based dyes for p-type dye-sensitized solar cells, Org. Lett. 13, 4930 (2011)

20. Y. Hong, J.-Y. Liao, D. Cao, et al., Organic dye bearing asymmetric double donor- $\pi$-acceptor chains for dye-sensitized solar cells, J. Org. Chem. 76, 8015 (2011)

21. F.-T. Kong, S.-Y. Dai, K.-J. Wang, Review of recent progress in dye-sensitized solar cells, Adv. Optoelectron. 2007, e75384 (2007)

22. Z. Liu, H. Ojima, Z. Hong, J. Kido, W. Tian, X.-F. Wang, Solution-processed organic photovoltaics based on indoline dye molecules developed in dye-sensitized solar cells, Mol. Basel. Switz. 18, 3107 (2013)

23. Sigma-Aldrich, Disperse Red 1, product number 344206 (2016) http://www.sigmaaldrich.com/catalog/product/ aldrich $/ 344206$ ?lang $=$ en\&region $=$ US

24. W.Y. Huang, P.T. Huang, Y.K. Han, C.C. Lee, T.L. Hsieh, M.Y. Chang, Aggregation and gelation effects on the performance of poly(3-hexylthiophene)/fullerene solar cells, Macromolecules 41, 7485 (2008)

25. H.N. Ghosh, A.V. Sapre, J.P. Mittal, Aggregation of C70 in solvent mixtures, J Phys. Chem. 100, 9439 (1996)
26. B. Watts, W.J. Belcher, L. Thomsen, H. Ade, P.C. Dastoor, A quantitative study of PCBM diffusion during annealing of P3HT:PCBM blend films, Macromolecules 42, 8392 (2009)

27. M. Reyes-Reyes, K. Kim, D.L. Carroll, High-efficiency photovoltaic devices based on annealed poly(3-hexylthiophene) and 1-(3-methoxycarbonyl)-propyl-1- phenyl- $(6,6)$ C61 blends, Appl. Phys. Lett. 87, 083506 (2005)

28. G. Li, V. Shrotriya, Y. Yao, Y. Yang, Investigation of annealing effects and film thickness dependence of polymer solar cells based on poly(3-hexylthiophene), J. Appl. Phys. 98, $043704(2005)$

29. H. Hoppe, N.S. ariciftci, Morphology of polymer/fullerene bulk heterojunction solar cells, J. Mater. Chem. 16, 45 (2006)

30. Q. Ying, J. Marecek, B. Chu, Slow aggregation of buckminsterfullerene (C60) in benzene solution, Chem. Phys. Lett. 219, 214 (1994)

31. R. Peng, J. Zhu, W. Pang, et al., Thermal annealing effects on the absorption and structural properties of regioregular poly (3-hexylthiophene) films, J. Macromol. Sci. Part B 50, 624 (2011)

32. X. Yang, J. Loos, Toward high-performance polymer solar cells: the importance of morphology control, Macromolecules 40, 1353 (2007)

33. I.E. Jacobs, F. Wang, Z.I.B. Valdez, A.N.A. Oviedo, D.J. Bilsky, A.J. Moulé, Photoinduced degradation from trace 1,8-diiodooctane in organic photovoltaics, J. Mater. Chem. C 6, 219 (2018)

34. E. Lewis, B. Mantha, R.P. Barber, Resistance and lifetime measurements of polymer solar cells using glycerol doped poly[3,4-ethylenedioxythiophene]: poly[styrenesulfonate] hole injection layers, EPJ Photovoltaics 5, 50402 (2014)

35. N.D. Treat, M.A. Brady, G. Smith, M.F. Toney, E.J. Kramer, C.J. Hawker, M.L. Chabinyc, Interdiffusion of PCBM and P3HT reveals miscibility in a photovoltaically active blend, Adv. Energy Mater. 1, 82 (2011)

36. E.L. Sena, J.H. Peel, D. Wesenberg, S. Nathan, M. Wallis, M. J. Giammona, T. Adalsteinsson, B.J. McNelis, R.P. Barber, Transport and spectroscopic studies of the effects of fullerene structure on the efficiency and lifetime of polythiophenebased solar cells, Sol. Energy Mater. Sol. Cells 100, 192 (2012)

Cite this article as: Kyle J. Bandaccari, Grace E. Chesmore, Mitchel Bugaj, Parisa Tajalli-Tehrani Valverde, Richard P. Barber Jr., Brian J. McNelis, Time-dependent efficiency measurements of polymer solar cells with dye additives: unexpected initial increase of efficiency, EPJ Photovoltaics 9, 4 (2018) 